\title{
Homologous Mechanisms of Visuospatial Working Memory Maintenance in Macaque and Human: Properties and Sources
}

\author{
Robert M. G. Reinhart, Richard P. Heitz, Braden A. Purcell, Pauline K. Weigand, Jeffrey D. Schall, \\ and Geoffrey F. Woodman \\ Department of Psychology, Vanderbilt Vision Research Center, Center for Integrative and Cognitive Neuroscience, Vanderbilt University, Nashville, \\ Tennessee 37240
}

\begin{abstract}
Although areas of frontal cortex are thought to be critical for maintaining information in visuospatial working memory, the event-related potential (ERP) index of maintenance is found over posterior cortex in humans. In the present study, we reconcile these seemingly contradictory findings. Here, we show that macaque monkeys and humans exhibit the same posterior ERP signature of working memory maintenance that predicts the precision of the memory-based behavioral responses. In addition, we show that the specific pattern of rhythmic oscillations in the alpha band, recently demonstrated to underlie the human visual working memory ERP component, is also present in monkeys. Next, we concurrently recorded intracranial local field potentials from two prefrontal and another frontal cortical area to determine their contribution to the surface potential indexing maintenance. The local fields in the two prefrontal areas, but not the cortex immediately posterior, exhibited amplitude modulations, timing, and relationships to behavior indicating that they contribute to the generation of the surface ERP component measured from the distal posterior electrodes. Rhythmic neural activity in the theta and gamma bands during maintenance provided converging support for the engagement of the same brain regions. These findings demonstrate that nonhuman primates have homologous electrophysiological signatures of visuospatial working memory to those of humans and that a distributed neural network, including frontal areas, underlies the posterior ERP index of visuospatial working memory maintenance.
\end{abstract}

\section{Introduction}

Working memory is the cognitive substrate that allows us to hold and manipulate information over relatively brief time intervals and is critical for complex human behavior (Baddeley, 2003). Although behavioral studies have often been used to infer the nature of the representations we store (Luck and Vogel, 1997; Cowan, 2001; Zhang and Luck, 2008; Perez and Vogel, 2012), the recent discovery of a human event-related potential (ERP) component that indexes the maintenance of information in visuospatial working memory has allowed for neuroscientific study of these representations, individual differences, and the role of working memory representations in attention tasks (Vogel and Machizawa, 2004; Vogel et al., 2005; Woodman and Vogel, 2008;

Received Jan. 13, 2012; revised March 29, 2012; accepted April 8, 2012.

Author contributions: J.D.S. and G.F.W. designed research; R.M.G.R., R.P.H., B.A.P.,P.K.W., and G.F.W. performed research; R.M.G.R., R.P.H., and B.A.P. contributed unpublished reagents/analytic tools; R.M.G.R. analyzed data; R.M.G.R., J.D.S., and G.F.W. wrote the paper.

This work was supported by National Institutes of Health Grants R01-EY019882, P30-EY08126, and P30HD015052, by National Science Foundation Grant BCS-0957072, and by Robin and Richard Patton through the E. Bronson Ingram Chair in Neuroscience. We thank John Haitas, Mary Feurtado, and Michelle Young for their technical assistance.

The authors declare no competing financial interests.

Correspondence should be addressed to Geoffrey F. Woodman, PMB 407817, 2301 Vanderbilt Place, Vanderbilt University, Nashville, TN 37240-7817. E-mail: geoffrey.f.woodman@vanderbilt.edu.

DOI:10.1523/JNEUROSCI.0215-12.2012

Copyright $\odot 2012$ the authors $\quad 0270-6474 / 12 / 327711-12 \$ 15.00 / 0$
Carlisle et al., 2011). This component is most commonly known as the contralateral delay activity (or CDA) and is characterized by a lateralized posterior potential that is sustained during the retention interval of a short-term memory task. In addition, recent studies of EEG oscillations have suggested that the CDA may be due to the suppression of posterior rhythmic activity in the alpha band, contralateral to the remembered stimulus (Mazaheri and Jensen, 2008, 2010; van Dijk et al., 2010). However, if we are going to understand the neuronal and postsynaptic mechanisms that give rise to this critical neuroscientific tool for studying temporary memory, we need to simultaneously record activity within the brain.

Research spanning spatial scales (e.g., from local to large-scale electrical fields) is one of the greatest needs for understanding cognitive brain dynamics (Nunez and Srinivasan, 2006). The absence of such research has limited our ability to definitively identify neural generators of ERP components given the ambiguities involved in inversely modeling neural sources (Helmholtz, 1853; Luck, 2005; Nunez and Srinivasan, 2006). One solution to this problem is to establish homology between ERP components observed in monkeys and humans and then record the postsynaptic activity in different brain regions of the monkey to determine whether these local fields contribute to the components of interest (Schroeder et al., 1991, 1992; Lamme et al., 1992; Woodman, 2010). The present study takes this underused approach to an age-old problem. 


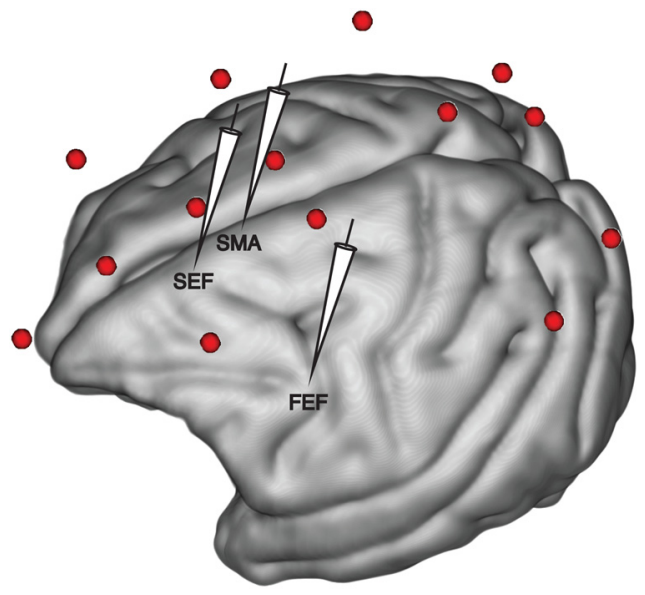

Figure 1. Electrode locations for simultaneous recording of intracranial LFPs and extracranial ERPs. Monkey LFP data were acquired from the FEF, SEF, and SMA. Monkey ERPs were acquired from cranial surface electrodes (red discs).

The memory-guided saccade task has been the modal working memory paradigm in neuroscience (Gnadt and Andersen, 1988; Funahashi et al., 1989; Colby et al., 1996; Constantinidis and Steinmetz, 1996; Chafee and Goldman-Rakic, 1998; Constantinidis et al., 2002; Wang et al., 2011). This task requires that a subject remember a particular spatial location over a delay period. We had both humans and macaque monkeys perform the same memory-guided saccade task to test the hypothesis that the CDA is found in both primate species. While recording EEG and ERPs from the monkeys using an array of surface electrodes, we simultaneously recorded local field potentials (LFPs) from the frontal eye field (FEF), the supplementary eye field (SEF), and the supplementary motor area (SMA) (Fig. 1). Although it is known that neurons in regions of frontal cortex exhibit sustained activity during the memory-guided saccade task, it is unknown whether these regions contribute to the generation of the decidedly more posterior CDA.

\section{Materials and Methods}

Subjects

We recorded surface ERPs and intracranial activity from four macaque monkeys. All procedures and care of the monkeys were performed with supervision and approval from the Vanderbilt Institutional Animal Care and Use Committee in accordance with the Public Health Service Policy on Humane Care and Use of Laboratory Animals. Human ERP data were collected from 10 neurologically normal subjects (age range, 19-30; six were females). Each participated in both Experiments 1 and 2 after we obtained informed consent. All procedures were approved by the Vanderbilt University Institutional Review Board.

\section{Experimental task and recording}

Monkeys and humans both performed the same memory-guided saccade task (Fig. 2, Experiment 1). Each trial began with subjects fixating a central $0.4^{\circ}$ square fixation point $(800-1200 \mathrm{~ms}$ duration randomly jittered with a rectangular distribution). A $1 \times 1^{\circ}$ circle target flashed alone for $100 \mathrm{~ms}$ at one of eight isoeccentric locations equally spaced $8-10^{\circ}$ around the fixation point. Trials with different target locations were randomly interleaved to maximize spatial uncertainty. The critical feature of this task is that subjects were required to maintain fixation for $500-1000 \mathrm{~ms}$ after target onset (i.e., the delay period or retention interval length; monkey S, 500 ms; monkeys F and Z, 600 ms; monkey Q, 750 ms; humans, $1000 \mathrm{~ms}$ ). Different lengths of delay periods were used across the macaques because of individual differences in their ability to remain on task with longer retention intervals. When the fixation point center extinguished, a $2 \mathrm{~s}$ window began in which subjects made a saccadic eye

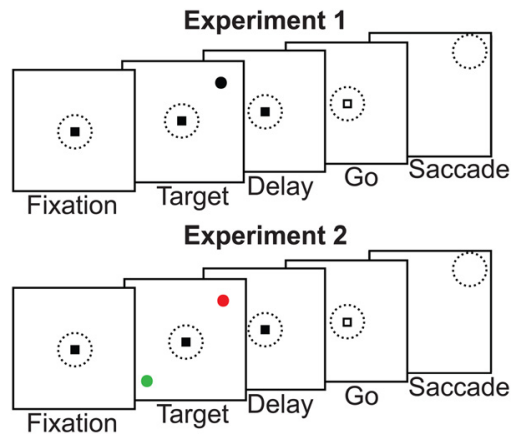

Figure 2. Schematic representation of the memory-guided saccade tasks. In Experiment 1, performed by both humans and monkeys, each trial began with subjects holding fixation on a central fixation point. A target appeared briefly at one of eight possible locations at random at an $8-10^{\circ}$ radius. The disappearance of the fixation point center cued subjects to make a memory-guided saccadic eye movement to the remembered spatial location. In Experiment 2 , performed only by humans, a two-stimulus hemifield-balanced memory array was used to disambiguate sensory evoked potentials from the CDA component. The target stimulus in Experiment 2 was either red or green, counterbalanced across subjects. The dotted circle indicates gaze position.

movement to the remembered location of the target, and then maintained gaze at that remembered location for 750-1000 ms. Monkeys were rewarded for correctly performed trials. For a response to count as qualitatively correct, the saccade needed to be made to a location within $2.5^{\circ}$ of the center of the memory target. All stimuli were isoluminant on a black background. The intertrial interval was $600 \mathrm{~ms}$ (1000 ms for humans and monkey Z). To precisely identify the onset of the CDA in humans, an additional memory-guided saccade task was devised (i.e., Fig. 2, Experiment 2). The difference between Experiments 1 and 2 was that the latter contained the presentation of one distractor stimulus in isoeccentric space directly opposite the location of the target. Target and distractor were distinguished by isoluminant colors (red and green), counterbalanced across human subjects. This second version of the memory-guided saccade task, with stimuli in both hemifields, allowed us to distinguish the CDA from lateralized sensory responses that are unrelated to working memory maintenance of the task-relevant information.

The FEF LFPs were recorded from both hemispheres of two male bonnet macaques (Macaca radiata; identified as Q, 11 years of age, $\sim 7 \mathrm{~kg}$; and S, 11 years of age, $\sim 8 \mathrm{~kg}$ ). SEF LFPs were recorded from the right hemisphere of one male bonnet macaque (identified as F, 18 years of age, $\sim 15 \mathrm{~kg}$ ). The SMA LFPs were recorded from the right hemisphere of one male rhesus macaque (Macaca mulatta; identified as Z, 8 years of age, $\sim 12.5 \mathrm{~kg}$ ). Simultaneously, we recorded ERPs from skull electrodes located at approximately OL/OR (in monkeys Q, S, and F), and FpFz, FCz, $\mathrm{Cz}, \mathrm{Pz}, \mathrm{POz}, \mathrm{Oz}, \mathrm{F} 3 / 4, \mathrm{C} 3 / 4$, T5/6, O1/2 (in monkey $\mathrm{Z}$ ) according to the human 10-20 system scaled to the macaque skull (Fig. 3) (Woodman et al., 2007; Godlove et al., 2011). LFPs were recorded from 2-5 M $\Omega$ impedance electrodes, sampled at $1 \mathrm{kHz}$, and filtered at $0.7-170 \mathrm{~Hz}$, using Plexon head-stage HST/8 050-G20 with an input impedance of $38 \mathrm{M} \Omega$. Monkey EEGs were sampled at $1 \mathrm{kHz}$ and filtered between 0.7 and 170 $\mathrm{Hz}$. A guide tube in contact with the surface of the dura was used as reference for the LFP signals. The frontal EEG electrode was used as reference for the EEG signals (approximately human $\mathrm{Fz}$ for monkeys Q, S, and F, and FpFz for monkey Z) (Woodman et al., 2007). Eye position was monitored using an eye coil or video-based infrared eye-tracking system (EyeLink; SR Research). Eye traces were recorded at $1 \mathrm{kHz}$. All surgical procedures were performed under aseptic conditions with general anesthesia.

Human EEG was recorded (250 Hz sampling rate; 0.01-100 Hz bandpass filter) using an SA Instrumentation amplifier connected to 21 tin electrodes, including 3 midline ( $\mathrm{Fz}, \mathrm{Cz}, \mathrm{Pz}), 8$ lateral pairs (F3/F4, C3/C4, P3/P4, PO3/PO4, T3/T4, T5/T6, O1/O 2 ), and 2 nonstandard sites: OL (midway between $\mathrm{O} 1$ and T5) and $\mathrm{OR}$ (midway between $\mathrm{O}_{2}$ and T6), arrayed according to the International 10-20 System and embedded in an elastic cap (Electro-Cap International). The right mastoid electrode 


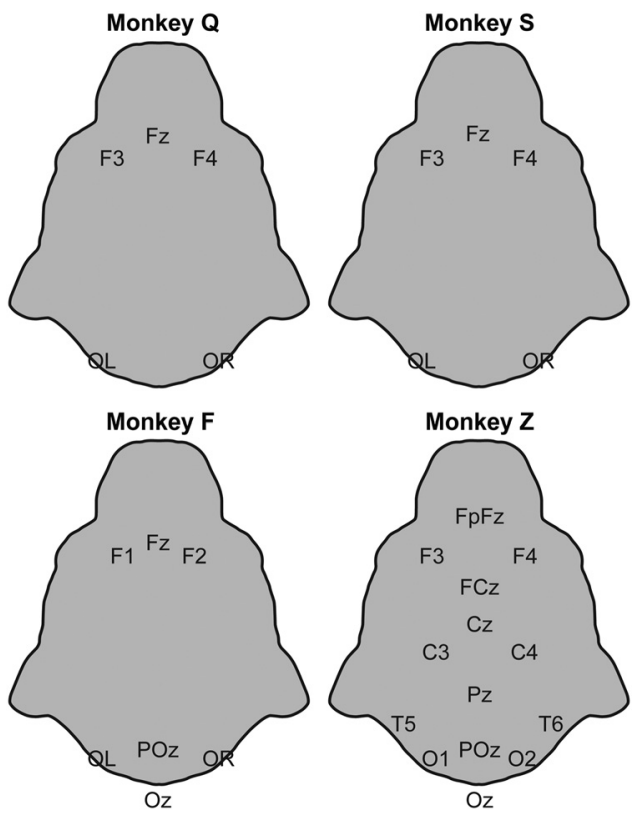

Figure 3. Monkey surface EEG electrode montages. Monkey $Q, S$, and $F$ were implanted with relatively sparse surface electrode configurations, whereas monkey $Z$ was implanted with a dense electrode array.

served as the on-line reference for these active electrode sites. Signals were rereferenced off-line to the average of the left and the right mastoids (Luck, 2005). Horizontal and vertical eye positions were monitored by recording electro-oculogram (EOG) from bipolar electrodes located at the outer canthi of each eye, and above and below the orbit of the left eye, respectively. All electrode impedances were kept under $5 \mathrm{k} \Omega$.

\section{Data analysis}

Behavior. A custom MATLAB function (MathWorks) automatically identified saccade initiation and termination using the EOG and eye tracker signals. This function measured when the instantaneous saccadic velocity became elevated above $30 \%$ and then calculated the beginning and end of the monotonic change in eye position (Hanes and Schall, 1995). To measure the precision of the saccadic responses beyond the coarse binary distinction of correct versus incorrect, we adopted the method for estimating variable saccade error as implemented in previous work (White et al., 1994), which has demonstrated that increasingly heavy memory demands in this spatial working memory task increase saccadic errors best captured with this metric. Briefly, saccade error estimates were based on measures of the amplitude and endpoint of the horizontal and vertical components of the first saccade after the eyes left central fixation. Vectorial eye velocity was computed from the horizontal and vertical eye position data using the central-difference differential algorithm (Bahill and McDonald, 1983). Trials with premature saccades to the memory stimulus or during the delay were excluded from analysis. The index of variable error (i.e., the scatter in saccadic endpoints) was obtained by first calculating the average horizontal and the average vertical eye position at the end of the initial saccade for each single target location (i.e., $0,45,90,135,180,225,270,315^{\circ}$ ) and for each human subject or monkey recording session. Then, for each saccade in this target by subject (or session) data matrix, the straight-line distance of each individual endpoint from the calculated average endpoint was obtained using the following formula:

$$
d_{i}=\sqrt{\left(\bar{X}^{\prime \prime} X_{i}\right)^{2}+\left(\bar{Y}^{\prime \prime} Y_{i}\right)^{2}}
$$

where $d_{i}$ is the deviation of saccade endpoint from the endpoint average, $\bar{X}$ is the average horizontal end position, $X_{i}$ is the horizontal endpoint for a particular saccade $i, \bar{Y}$ is the average vertical end position, and $Y_{i}$ is the vertical end position for saccade $i$. Finally, we computed a Pearson correlation coefficient between mean variable saccade endpoint error and mean CDA amplitude across trials for each human subject or each recording session from a monkey.

Electrophysiology: amplitudes and onsets. Grand average target-locked contralateral versus ipsilateral waveforms were generated collapsed across left and right hemispheres across our electrophysiological measures. Specifically, ERPs from the human subjects were averaged across subjects $(N=10)$, ERPs from the macaques were averaged across recording sessions ( $\mathrm{Q}, 109 ; \mathrm{S}, 31 ; \mathrm{F}, 33 ; \mathrm{Z}, 39)$, and LFPs from monkeys averaged across recording sites $(\mathrm{Q}, 38 ; \mathrm{S}, 62 ; \mathrm{F}, 66 ; \mathrm{Z}, 78)$. Waveforms were baseline corrected to the average of the activity in the $200 \mathrm{~ms}$ window preceding target onset. Trials were collapsed across the left and right hemifield stimulus conditions (left: $45,90,135^{\circ}$; right: $225,270,315^{\circ}$ ) to increase the power brought to bear. The number of trials at each target location was matched by excluding random trials from the overrepresented target locations. Single-trial target-locked EEG and LFP epochs were truncated $20 \mathrm{~ms}$ before saccade onset to eliminate artifacts arising from possible temporally smeared saccade activity. Trials in which subjects failed to execute the correct eye movement within the allotted time were excluded from the electrophysiological analyses and counted as incorrect.

The CDA mean amplitude was measured at lateral occipital ERP electrode sites as the difference in mean amplitude between the contralateral and ipsilateral waveforms using conservative measurement windows based on approximate mean latency onset and delay interval end time (humans, 450-1000 ms; monkey S, 350-500 ms; monkey Q, 350-750 $\mathrm{ms}$; monkeys $\mathrm{F}$ and $\mathrm{Z}, 350-600 \mathrm{~ms}$ ). The same method was used to calculate LFP polarization measured within FEF, SEF, and SMA (monkey S, 300-500 ms; monkey Q, 300-750 ms; monkey F and Z, 300-600 ms). Surface CDA and mnemonic LFP latency onsets were defined by the following significance test. First, a difference wave was constructed by subtracting ipsilateral from contralateral ERPs. The variability of the difference wave was assessed by calculating the SD during the baseline period ( -200 to $0 \mathrm{~ms}$ ). Significant epochs (shown with gray-shaded zones in the figures) were defined as periods when the difference wave deviated from baseline by $>2$ SDs for $>50 \mathrm{~ms}$, provided it exceeded 3 SDs in that interval. These are the same analytical methods used in previous simultaneous recordings of surface ERPs and intracranial LFPs (Cohen et al., 2009).

The above-mentioned CDA measurement is based on a common approach for calculating the human CDA component, namely a comparison between targets in the hemifield contralateral versus ipsilateral to the EEG electrode (Perez and Vogel, 2012). However, for the monkeys, we also calculated a comparison based on the receptive field (RF) of the neuron recorded simultaneously with the ERPs and LFPs (as in Cohen et al., 2009). We measured the difference between the ERPs and LFPs on trials when the target stimulus was inside the RF of the neuron and the ERPs and LFPs on trials when the target fell at the location(s) opposite the $\mathrm{RF}$ of the neuron. ANOVAs were used for statistical tests of amplitude and latency, and $p$ values were adjusted using the Greenhouse-Geisser $\varepsilon$ correction for nonsphericity, where appropriate (Jennings and Wood, 1976). For our correlation analyses relating the electrophysiological findings to behavior, we recalculated the correlations while excluding outliers ( $>2$ SDs). For the correlation coefficients reported, the same pattern of results was obtained. That is, the significant correlations that are reported were significant with or without outliers excluded.

Electrophysiology: spatial distributions. The spatial distribution of current densities producing the CDA was computed for all human subjects and monkey $\mathrm{Z}$ (i.e., the animal implanted with the highest density of surface electrodes) using all of the electrodes across the head, including frontal sites. These methods were identical with previous analyses of the spatial distributions of other ERP components observed across primate species (Godlove et al., 2011; Reinhart et al., 2012). ERP data from monkey $\mathrm{Z}$ were coregistered spatially with anatomical magnetic resonance images (MRIs) to enable current density analysis using the multimodal neuroimaging software CURRY 6 (Compumedics Neuroscan). While under sedation, the monkey was placed in a stereotaxic apparatus customized for the MRI environment (Crist Instrument). Anatomical MRIs were acquired with a Philips Intera Achieva 3 tesla scanner using a SENSE Flex-S two-element phased array receive only coil. One element was placed below the head and the other at the skull vertex. T1-weighted 
A

Humans Experiment 1

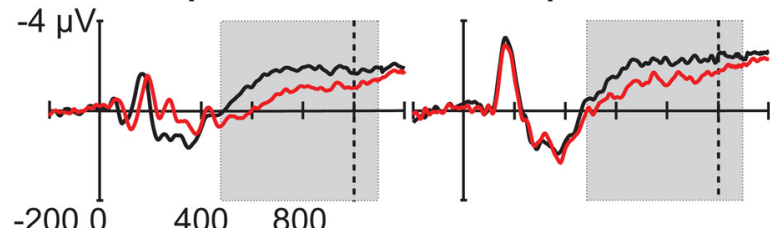

Time from Target Onset (ms)

Humans Experiment 2
C

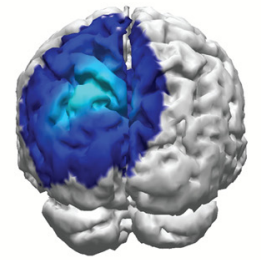

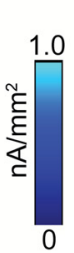

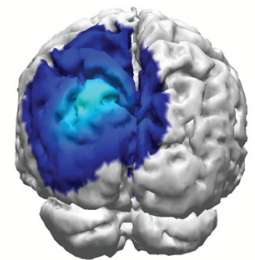

B Monkeys Q \& S

Monkeys F \& Z

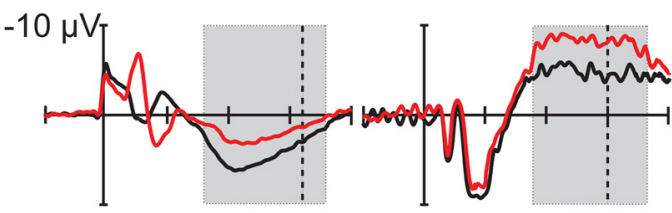

D

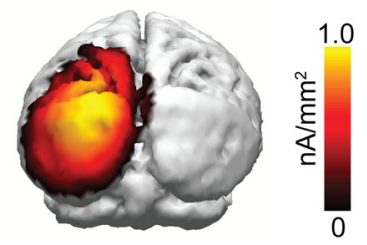

Figure 4. Human and monkey CDA waveforms and spatial distributions recorded from the surface EEG electrodes. Grand average target-locked ipsilateral (red) versus contralateral (black) waveforms are illustrated for Experiments 1 and 2 in humans $(\boldsymbol{A})$, and for Experiment 1 in monkeys $(\boldsymbol{B})$. Plotted with negative voltage up and time relative to the onset of the memory item. The waveforms were collapsed across target locations (left: $45,90,135^{\circ}$; right: $225,270,315^{\circ}$ ) and across left and right hemisphere occipital electrode sites. The gray-shaded regions highlight the period during which ipsilateral and contralateral waveforms relative to the remembered location significantly diverged, defining the CDA component. The dashed vertical line marks the time subjects were cued to respond. Current density distributions derived from the grand average CDA differences waves (ipsilateral minus contralateral) are shown for Experiments 1 and 2 in humans $(\boldsymbol{C})$ and for Experiment 1 in monkey $Z$, who was implanted with a dense electrode array $(\boldsymbol{D})$. The color scale difference highlights the polarity reversal in CDA between species. The lighter colors indicate greater current density with distributions shown for right visual field memory items.
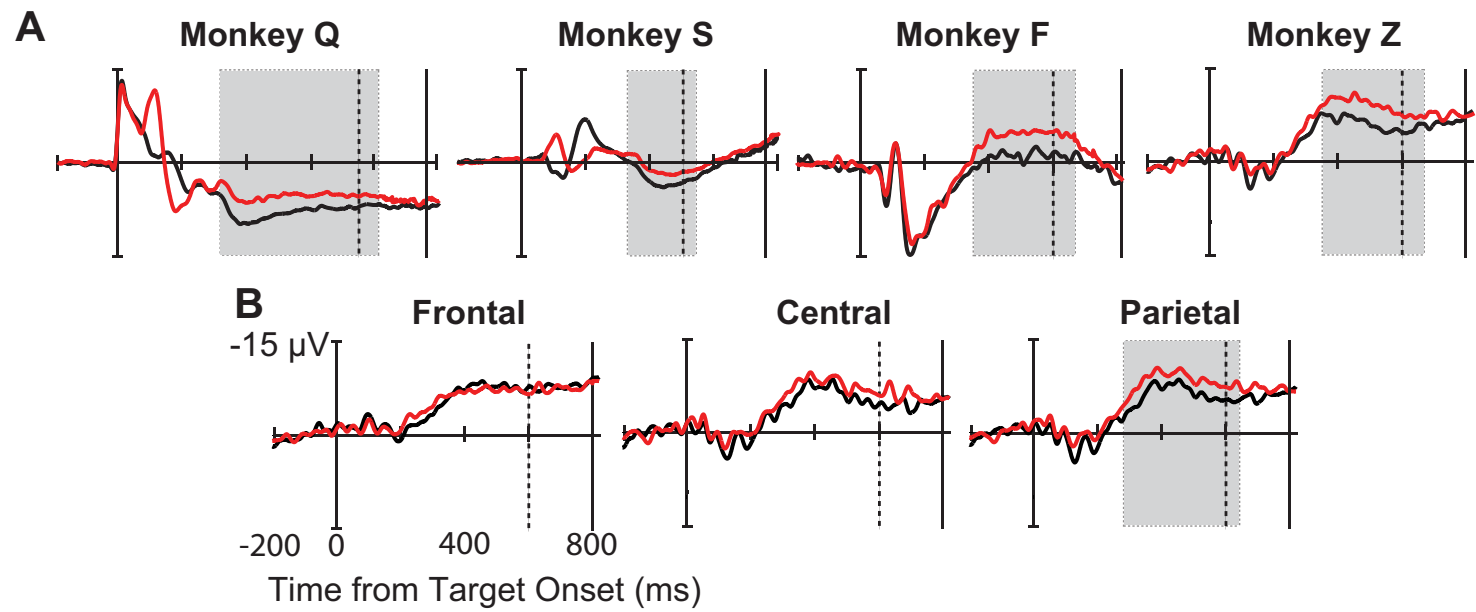

Figure 5. Surface ERP waveforms from each individual monkey. $A$, Grand average ERPs time-locked to the memory-target onset. Ipsilateral (red) versus contralateral (black) ERP waveforms from lateral occipital electrodes are illustrated for each macaque monkey. B, ERP waveforms across frontal, central, and parietal lateral electrode pairs from monkey Z. The gray-shaded zones indicate the period during which ipsilateral and contralateral waveforms significantly diverged (i.e., CDA component). The broken vertical line marks the time subjects were cued to respond, and the solid black vertical line indicates median saccadic reaction time. Monkey Q's early sensory evoked potentials were contaminated by a CRT monitor artifact, resulting in a $0 \mathrm{~ms}$ latency of the visual response. However, monkey Q exhibited a significant surface CDA with similar onset latency, amplitude, correlation to behavioral performance, and correspondence to the LFP polarization during the memory retention interval (Fig. 12). Furthermore, monkey $Q$ exhibited significant delay period rhythmic oscillations recorded intracranially (Fig. 12E) and extracranially (Fig. 10).

gradient-echo structural images were obtained with a $3 \mathrm{D}$ turbo field echo anatomical sequence (TR, $8.729 \mathrm{~ms} ; 130$ slices; $0.70 \mathrm{~mm}$ thickness). A high-resolution 3D model of the segmented skull and brain was constructed in CURRY 6. The cranial surface electrode locations were coregistered to the head model guided by stereotaxic coordinates recorded during surgery. From this 3D head model, a three-compartment boundary element method (BEM) volume conductor geometry was generated. The BEM model consisted of 2704 triangle meshes overall, or 1358 nodes, which describe the smoothed inner skull, the outer skull, and the outside of the skin. The mean triangle edge lengths (node distances) were $9 \mathrm{~mm}$ (skin), $8 \mathrm{~mm}$ (skull), and $6 \mathrm{~mm}$ (brain compartment). Standard conductivity values for the three compartments were used as follows: skin, 0.33 $\mathrm{S} / \mathrm{m}$; skull, $0.0042 \mathrm{~S} / \mathrm{m}$; brain, $0.33 \mathrm{~S} / \mathrm{m}$. Of note, CURRY is designed to provide solutions from skin surface electrodes; however, we measured monkey ERPs from the cranial surface. Skin conductance and thickness values did not contribute to the computed model solutions in monkeys because electrodes were located on the skull surface.

For humans, the interpolated BEM model was derived from averaged MRI data from the Montreal Neurological Institute. It consisted of 9300 triangular meshes overall, or 4656 nodes, with mean triangle edge lengths of $9 \mathrm{~mm}$ (skin), $6.8 \mathrm{~mm}$ (skull), and $5.1 \mathrm{~mm}$ (brain compartment). Standard conductivity values for the three compartments were set to the following: skin, $0.33 \mathrm{~S} / \mathrm{m}$; skull, $0.0042 \mathrm{~S} / \mathrm{m}$; and brain, $0.33 \mathrm{~S} / \mathrm{m}$. The interpolated BEM model was built using the onboard CURRY 6 MRI dataset (Fuchs et al., 2002).

The current density analysis was calculated from the voltage difference waves (contralateral minus ipsilateral) during the memory retention interval for humans (450-1000 ms) and monkey Z (350-600 ms). Current 


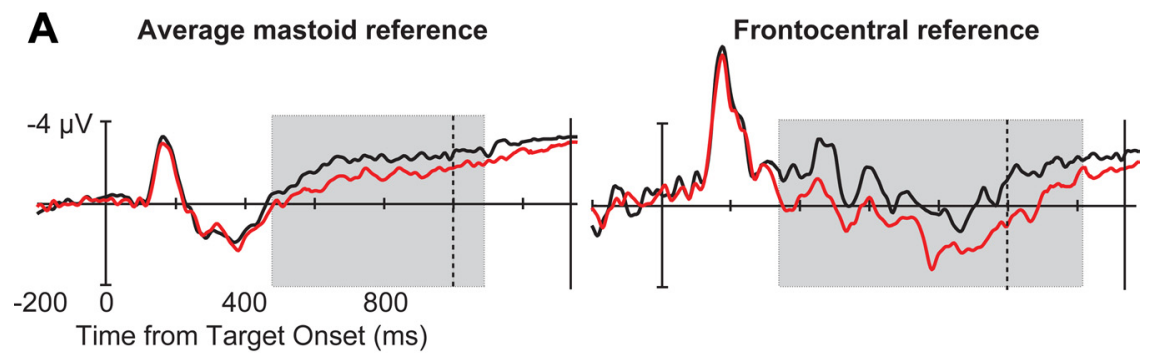

B
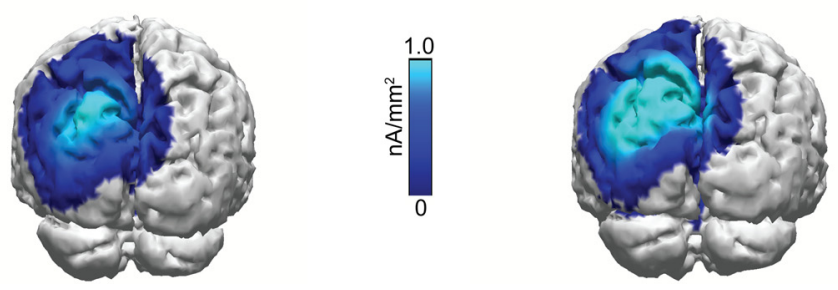

Figure 6. Human CDA waveforms and spatial distributions across reference electrode configurations. $\boldsymbol{A}$, Grand average targetsynchronized ipsilateral (red) versus contralateral (black) ERP waveforms are shown using an average mastoid reference electrode configuration (left panel), the standard in human CDA research, as well as a frontocentral reference electrode configuration similar to that of each monkey's ERPs in the present study (right panel). The gray-shaded zones indicate the period during which ipsilateral and contralateral waveforms significantly diverged (i.e., CDA component). The broken vertical line marks the time subjects were cued to respond, and the solid black vertical line indicates median saccadic response time. $\boldsymbol{B}$, Current density distributions derived from the grand average CDA difference waves (contralateral minus ipsilateral) are illustrated for humans with both reference electrode sites. The lighter colors indicate greater current density with distributions shown for right visual field memory items. obtaining complex time-frequency data points for every individual trial, these data were transformed into a total power measure. For each frequency in the resulting time-frequency matrix, the baseline period was defined by the average of the values within a -200 to $0 \mathrm{~ms}$ time window preceding the time-locking target event. A simple subtraction of baseline values from all the values in the epoch was performed, as this is the most common approach to baseline correction in EEG and ERP research. An average of total power, single-trial values was calculated using conservative measurement windows based on approximate mean latency onset and delay interval end time, just as with the CDA (humans, 300-1000 ms; monkey S, 200-500 ms; monkey Q, 200-750 ms; monkeys $\mathrm{F}$ and Z, 200-600 ms). As with the CDA, figures were generated from measurements at lateral occipital electrode sites for EEG, and at lateral FEF, SEF, and SMA sites for LFPs as the difference in mean amplitude between the contralateral and ipsilateral waveforms. Similarly, we statistically compared the power in left and right target conditions for each hemisphere intracranially at theta $(4-8 \mathrm{~Hz})$ and gamma frequencies $(30-50 \mathrm{~Hz})$, and extracranially for electrodes sites OL and OR at the alpha and low-beta frequencies $(8-16 \mathrm{~Hz})$.

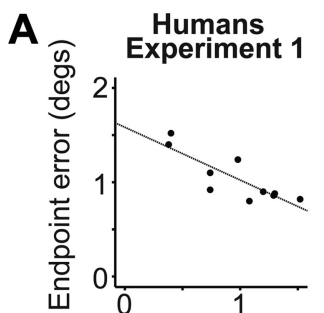

B

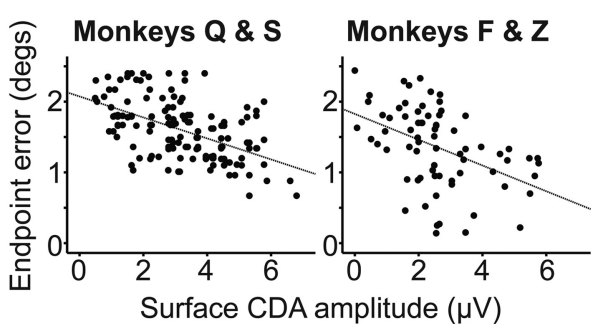

Figure 7. The relationship between human and monkey surface CDA amplitude and behavioral performance. Scatterplots of significantly negative linear correlations between mean variable error (in degrees of visual angle) and mean surface CDA amplitude (contralateral minus ipsilateral) are shown for Experiments 1 and 2 in humans $(\boldsymbol{A})$ and for Experiment 1 in monkeys $(\boldsymbol{B})$.

density was estimated using the standardized low-resolution electromagnetic tomography-weighted accurate minimum norm method (SWARM) (Wagner et al., 2007). For monkey Z, SWARM was estimated using the cranial surface electrode locations mentioned above, whereas for humans SWARM was estimated using electrode positions based on the International 10-20 System and a cortical surface obtained from a segmentation of the CURRY 6 individual reference brain.

Electrophysiology: time-frequency analyses. Time-frequency analyses were performed using a Morlet wavelet decomposition with FieldTrip software (Oostenveld et al., 2011). The Morlet wavelet has a Gaussian envelope that is defined by a constant ratio $\left(\sigma_{f}=f / 7\right)$ and a wavelet duration $\left(6 \sigma_{t}\right)$, where $f$ is the center frequency and $\sigma_{t}=1 /\left(2 \pi \sigma_{f}\right)$. After

\section{Results}

Behavior

Using the modal memory-guided saccade task in the neuroscience literature (Gnadt and Andersen, 1988; Funahashi et al., 1989; Colby et al., 1996; Constantinidis and Steinmetz, 1996; Chafee and Goldman-Rakic, 1998; Constantinidis et al., 2002; Wang et al., 2011), we found that monkeys and humans performed at similar levels of accuracy ( $>90 \%$ correct across all target locations and subjects, with the exception of monkey $\mathrm{S}$, whose percentage correct was $>75 \%$ correct) (for accuracy criteria, see Materials and Methods). For the qualitatively correct memory-guided saccades made in the vicinity of the memory target, the saccadic endpoint error for monkeys was $1.5 \pm 0.5^{\circ}$ (mean $\pm \mathrm{SD}$ ) and for humans was $1.0 \pm 0.3^{\circ}$ (Experiment 1 ) and $1.2 \pm 0.3^{\circ}$ (Experiment 2). Reaction times for monkeys were $248 \pm 31 \mathrm{~ms}$ (mean \pm SD) and for humans $318 \pm 58 \mathrm{~ms}$ (Experiment 1 ) and $320 \pm 51 \mathrm{~ms}$ (Experiment 2) across correct trials. Within species, the Pearson correlation between saccadic reaction times and positional error was not significant (monkey: $r_{(211)}=-0.025, p=0.891$; human Experiment $1: r_{(9)}=-0.189$, $p=0.301$; human Experiment 2: $r_{(9)}=-0.110, p=0.349$ ). Between species, a two-tailed $t$ test revealed significant differences between saccadic reaction times $(p<0.01)$ and positional error $(p<0.01)$ due to monkeys being faster but less accurate than humans. This pattern of memory-guided saccade performance is consistent with previous findings from humans (Curtis et al., 2004) and monkeys (Funahashi et al., 1989).

\section{Surface event-related potentials and oscillatory activity}

In humans, a sustained contralateral negativity arose $\sim 450 \mathrm{~ms}$ after memory target presentation over posterior electrode sites and continued through the memory retention interval (Fig. 4A). This is the primary defining characteristic of the CDA during change detection tasks in humans (Vogel and Machizawa, 2004; Vogel et al., 2005; Perez and Vogel, 2012). To determine which 


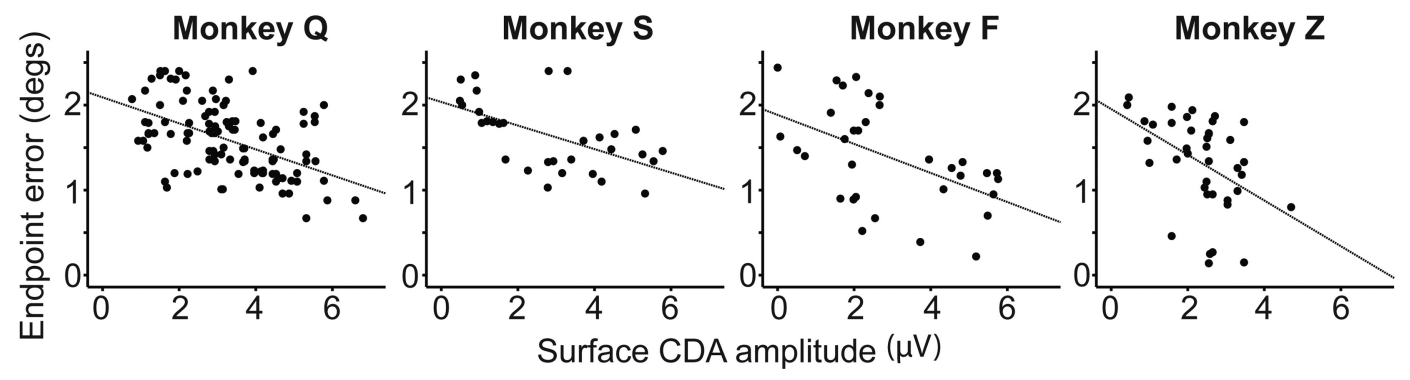

Figure 8. The relationship between each monkey's surface CDA and behavioral performance. Scatterplots showing significant negative linear correlations between mean variable saccadic endpoint error and mean surface CDA amplitude (ipsilateral minus contralateral waveforms) are illustrated for each monkey $(Q: r=-0.515, p<0.001 ; S: r=-0.564, p<0.001 ; \mathrm{F}: r=-0.506$, $p<0.01 ; z: r=-453, p<0.01)$.

portions of the waveforms were due to the potentially confounding effects of lateralized sensory evoked potentials, and verify the latency of the CDA in humans, the same group of human subjects performed a second memory-guided saccade task in which a distractor was presented directly opposite the task-relevant memory target in the other hemifield (Fig. 2, Experiment 2). Experiment 2 confirmed the onset of the human CDA during this spatial memory task was at $450 \mathrm{~ms}$ after stimulus (Fig. $4 A$ ).

In macaques, we found that the CDA, defined by the sustained difference in lateralized potentials, began at $\sim 350 \mathrm{~ms}$ after the memory target and took the form of a strong contralateral positivity over posterior electrodes. As in humans, this activity was sustained throughout the delay period (Fig. 4B). All monkeys exhibited a clear CDA during the memory retention interval following the initial sensory ERPs (Fig. 5). For monkeys Q and S, this sustained contralateral positivity rode on top of a bilateral positivity, whereas for monkeys $\mathrm{F}$ and $\mathrm{Z}$ this contralateral positivity appears to overlap with a bilateral negativity. Previous studies of the CDA in humans (Vogel and Machizawa, 2004; Carlisle et al., 2011) have shown that the CDA commonly overlaps with both the $\mathrm{P} 3$ component that is believed to index working memory updating (Donchin and Coles, 1988) and it is known to exist in nonhuman primates (Arthur and Starr, 1984) and later negativity slow waves that have been documented in working memory tasks (Ruchkin et al., 1992). It appears that, across monkeys, we are observing some individual differences in the onset and duration of these overlapping ERP components. Such individual differences are common in human ERP studies, although the source of such variation across subjects is not yet well understood (Woodman, 2010). It is notable that monkey F exhibited a CDA that was similar to the other macaques, given that this monkey was $\sim 18$ years of age. This finding appears to challenge a recent report in which the working memory-related activity of older macaque monkeys appears to be abnormal (Wang et al., 2011).

As is evident, the CDA latency differed by $\sim 100 \mathrm{~ms}$ between species (Fig. 4). However, a substantial portion of this interspecies latency difference is due to the use of different reference electrodes. A reanalysis of the human data using a frontocentral reference electrode, as in the monkey recordings, essentially eliminated this latency discrepancy (Fig. 6). However, when comparing the latency of the CDA in humans and one monkey (i.e., Z) rereferenced to similar mastoid reference sites, it appears that the monkey CDA does onset $\sim 75 \mathrm{~ms}$ earlier than the same effect in humans. This latency difference would be similar to previous studies of macaque ERPs in which onset latencies of homologous components are often $\sim 25 \%$ shorter than those observed in humans (Woodman, 2012). The interspecies difference in CDA polarity was reliably observed using both the standard average
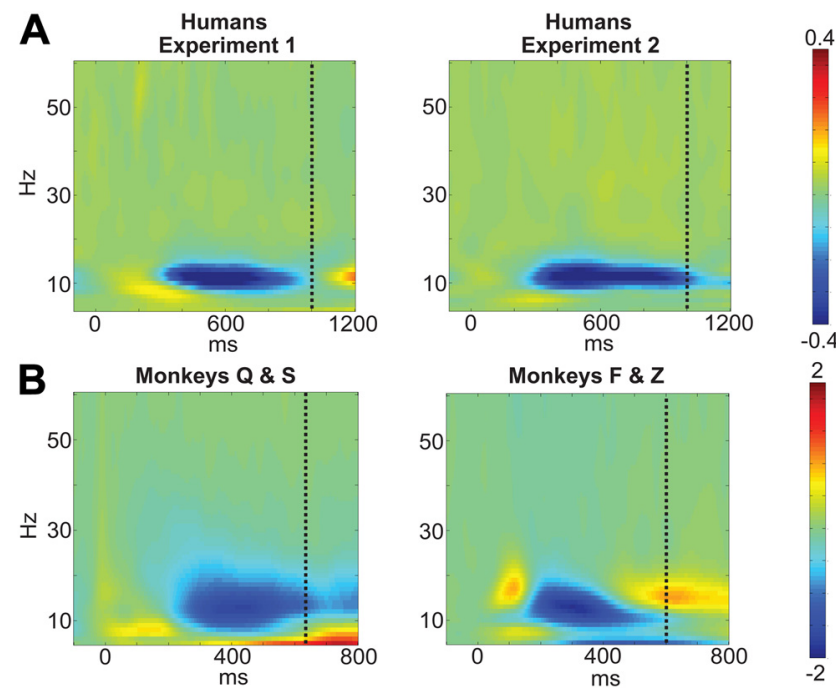

Figure 9. Time-frequency plots of lateralized surface total power. Grand average data are time-locked to the target stimulus and by convention shown as the difference between power for right and left hemifield stimuli collapsed across right and left hemisphere electrodes for human Experiments 1 and $2(\boldsymbol{A})$ and monkeys $(\boldsymbol{B})$. The dashed vertical line marks the time subjects were cued to respond. The plots show the contralateral suppression of power in the alpha band.

mastoid and frontocentral reference electrodes. This polarity inversion of homologous ERP components across species is consistent with previous studies of lateralized attention effects (Woodman et al., 2007; Cohen et al., 2009), but was not found for the error-related negativity and positivity in monkeys (Godlove et al., 2011). Such polarity inversions are most likely due to variation of cortical folding across species (Woodman, 2010) because the orientation of the cortical tissue generating the electrical fields measured in ERP recordings determines the polarity of the component. For example, in humans it appears that the same ERP component (i.e., the $\mathrm{C} 1$, which begins at $\sim 50 \mathrm{~ms}$ after stimulus) will flip polarity when the stimulus activates the inferior versus superior banks of the calcarine sulcus (Clark et al., 1995). Nevertheless, compared with the human, the monkey CDA exhibited a comparable latency following lateralized sensory transients and identical persistence throughout the memory retention interval. In both species, the CDA continued until the saccadic response was initiated. However, the lateralized ERPs did not exhibit an increase in amplitude immediately before the saccade, as would be expected if it reflected saccade preparation (Figs. 4, 5, 6A).

Like all ERP components, the current sources of the CDA can be modeled from the spatial distribution of voltages on the sur- 

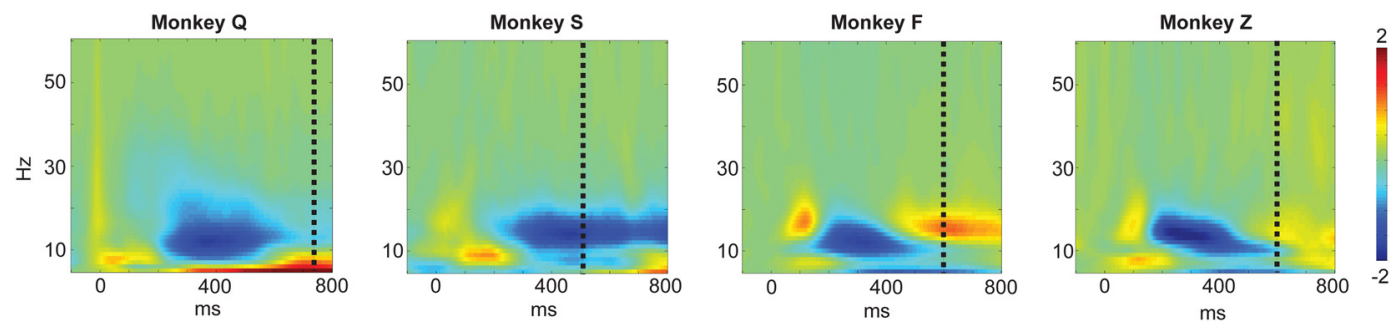

Figure 10. Individual monkey time-frequency plots of lateralized total power at the posterior surface electrodes. Grand average contralateral data are time-locked to the target stimulus and shown as the difference between power for right and left hemifield stimuli collapsed across right and left hemisphere electrodes for each monkey. The dashed vertical line marks the time subjects were cued to respond.

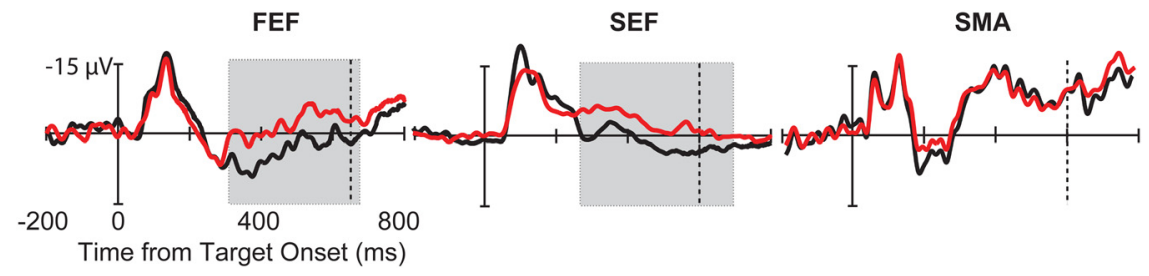

Figure 11. Monkey intracranial LFPs averaged relative to the remembered location. Grand average ipsilateral (red) versus contralateral (black) LFP waveforms relative to the remembered location are shown from recordings sites in FEF, SEF, and SMA. The waveforms were collapsed within left and right target locations (left: $45,90,135^{\circ}$; right: $225,270,315^{\circ}$ ) and across recording sites. The gray-shaded zones indicate the period during which ipsilateral and contralateral waveforms significantly diverged, and the broken vertical line marks the time subjects were cued to respond.

face using all of the electrodes on the head. The spatial distribution of the human CDA component from Experiments 1 and 2 was assessed during the epoch that a significant CDA was found (i.e., $450-1000 \mathrm{~ms}$ ). The dense electrode array of monkey $\mathrm{Z}$ allowed for the same analysis of the monkey CDA (from 350 to 600 ms) (Fig. 3 shows electrode configurations for each monkey). Distributed current densities were projected onto 3D reconstructions of anatomical MRIs from the monkey and a reference brain for humans. Current density in humans was concentrated in dorsal posterior foci explaining 97\% (Experiment 1) and 98\% (Experiment 2) of the variance of the surface potential distribution (Fig. 4C). Similarly, current density for the monkey CDA was concentrated in dorsal posterior areas (96\% explained variance) (Fig. $4 D$ ). We verified that a dorsal posterior scalp topography was also found in humans when using a frontocentral reference, as was used in the monkeys (Fig. $6 B$ ). Thus, the spatial distribution of current density producing the CDA in monkeys mirrored that of humans performing the memory-guided saccade task with a target presented alone or with a distractor.

A key advantage of the memory-guided saccade task is that it provides a graded metric of accuracy through the distribution of saccadic endpoints. Behavioral studies of the memory-guided saccade task in both humans (Ploner et al., 1998) and monkeys (White et al., 1994) have shown that measurements of saccadic endpoint scatter (i.e., the squared variability of saccadic endpoint, sometimes known as variable error) is more directly related to visual working memory maintenance than coarser measures of correct versus incorrect, or absolute error. To determine the relationship between the delay period activity and the fidelity of the mnemonic representation guiding the behavioral response, we tested the prediction that the amplitude of the CDA measured during the retention interval predicted the spatial precision of the subsequent memory-guided saccade. If the CDA provides a measure of the quality of the memory representation of the location, then we would expect that a larger CDA would result in less error due to a higher fidelity working memory representation. In contrast, if the CDA were simply measuring the eccentricity of the remembered location, then the CDA would not predict the accuracy of the behavioral report of the location, but instead simply the amplitude of the saccadic response, regardless of what the memory target location was.

We found that the mean amplitude of the CDA was predictive of the mean endpoint error using subjects as the unit of analysis for humans and recording sessions for the monkey data (Fig. 7). Significant negative correlations were observed between mean CDA amplitude and magnitude of saccadic error in humans (Experiment 1: $r_{(9)}=-0.841, p<0.01$; Experiment 2: $\left.r_{(9)}=-0.810, p<0.01\right)$ and monkeys $\left(\mathrm{Q}\right.$ and $\mathrm{S}: r_{(139)}=-0.528$, $p<0.01 ; \mathrm{F}$ and $\mathrm{Z:} r_{(71)}=-0.447, p<0.01$ ) (Fig. 8). In contrast, we found that the amplitude of the CDA did not strongly or reliably predict the eccentricity of the saccadic responses made in humans (values of $r=-0.06$ to -0.35 ; values of $p>0.17$ ) or monkeys (values of $r=-0.02$ to -0.24 ; values of $p>0.06$ ). We found a similar pattern of results when within-subject correlations were performed. Specifically, within-subject correlations mirrored those obtained using both measures of saccadic error (human and monkey: values of $p<0.01$ ) and saccadic amplitude (human: values of $p>0.11$; monkey: values of $p>0.10$ ). Thus, as predicted above, when CDA amplitude was higher, response error was lower.

It has recently been shown that systematic modulation of the lateralized posterior alpha rhythm $(8-13 \mathrm{~Hz})$ in humans may account for the slow and sustained evoked responses of the CDA (Mazaheri and Jensen, 2008, 2010; van Dijk et al., 2010). This hemisphere-specific lateralized EEG alpha activity over posterior cortex is often referred to as contralateral alpha-band suppression and is found during visuospatial working memory tasks in humans (Jokisch and Jensen, 2007; Van Der Werf et al., 2008; Grimault et al., 2009; Sauseng et al., 2009). To provide additional evidence that the macaque homolog of the human CDA is due to the same EEG dynamics, we spectrally decomposed EEG signals from both species to assess oscillatory fluctuations in the alpha band. Beginning $\sim 300 \mathrm{~ms}$ after memory target onset, alpha power in human was suppressed over parietooccipital electrodes in the hemisphere contralateral to the remembered stimulus (Fig. $9 A$ ). That is, when the target was presented in the right hemifield, the right electrodes showed significantly greater alpha-band power relative to left electrodes $\left(t_{(9)}=4.844 ; p<0.002\right)$. The same pattern of activity was observed for targets presented on the left $\left(t_{(9)}=2.691 ; p<0.03\right)$. These results replicate previous work 
(Jokisch and Jensen, 2007; Van Der Werf et al., 2008; Grimault et al., 2009; Sauseng et al., 2009) and demonstrate a strong lateralization in human alpha-band activity with respect to the hemifield of the remembered stimulus during memory retention.

In macaques, we found a contralateral alpha-band suppression beginning at $\sim 150 \mathrm{~ms}$ following memory target onset over posterior electrodes mirroring the EEG effects found in humans (Fig. $9 B$ ). All monkeys exhibited suppressed alpha and low-beta power at posterior electrodes in the hemisphere contralateral to the remembered stimulus. This resulted in power being significantly greater for the hemisphere ipsilateral to the remember stimulus (left hemisphere Q: $t_{(108)}=2.572, p<0.01 ; \mathrm{S}: t_{(30)}=3.158, p<0.004 ; \mathrm{F}: t_{(32)}=2.214$, $p<0.04$; Z: $t_{(38)}=2.613, p<0.01$; right hemisphere Q: $t_{(108)}=$ $2.704, p<0.009 ; \mathrm{S}: t_{(30)}=3.252, p<0.003 ; \mathrm{F}: t_{(32)}=2.076, p<0.05$; $Z: t_{(38)}=2.287, p<0.02$; Fig. 10). Despite the interspecies difference in CDA polarity previous noted, hemispheric lateralization of alpha power was reliably observed across species, providing further evidence that the human and monkey CDAs are indexing the same fundamental neural dynamics. In sum, time-frequency analysis of oscillatory power for both human and nonhuman primates revealed strong modulation of alpha-band activity during the delay period.

\section{Intracranial event-related local field potentials and oscillatory activity}

Although estimating the current density producing the CDA provides useful information, simultaneous intracranial recordings of LFPs are critical to identify the sources in the distributed network believed to underlie ERP components. This is because it is believed that the surface ERPs are generated by a spatially weighted average of the postsynaptic LFP activity in the brain, although a number of these sources might not contribute to the spatial distribution of a component due to cancellation or superposition (Helmholtz, 1853; Luck, 2005; Nunez and Srinivasan, 2006; Woodman, 2010). In macaque monkeys performing the memory-guided saccade task, the LFPs recorded from FEF and SEF, but not SMA, exhibited sustained delay period activity when the memory target was in the contralateral hemifield (Fig. 11) (see also Fig. 12A). This resulted in significantly more positive LFPs when the remembered location was contralateral to the site in $\operatorname{FEF}\left(F_{(1,99)}=15.280 ; p<0.01 ; 94 \%\right.$ of sites significant $)$ and $\operatorname{SEF}\left(F_{(1,65)}=22.160 ; p<0.01 ; 92 \%\right.$ of sites significant $)$, but not in SMA $\left(F_{(1,77)}=0.637 ; p=0.427 ; 4 \%\right.$ of sites significant $)$. In addition to the traditional approach of measuring the CDA component by comparing polarization when remembered items were presented in the contralateral versus the ipsilateral hemifield, we restricted the analysis to the trials when the memory target was located within the RF of the neuron recorded simultaneously with the LFPs. This more restrictive analysis yielded the same pattern of results with significant differences in the amplitude of the delay period LFPs when the memory item was in versus out of the $\mathrm{RF}$ of the neurons in $\operatorname{FEF}\left(F_{(1,99)}=13.770 ; p<0.01 ; 92 \%\right.$ of sites significant $)$ and $\operatorname{SEF}\left(F_{(1,65)}=17.738 ; p<0.01 ; 93 \%\right.$ of sites significant), but not in $\operatorname{SMA}\left(F_{(1,77)}=0.116 ; p=0.734 ; 1 \%\right.$ of sites significant).

Next, we examined how the sustained LFPs were related to behavioral accuracy in the visuospatial working memory task, just as we had with the CDA. Figures $12 B$ and 13 show that the amplitude of the LFPs in FEF and SEF predicted the precision of the subsequent behavioral report of the memory item at the end of the trial. Specifically, we found significant negative correlations between the mean amplitude of the LFP during the delay period (FEF, 300-500 ms; SEF, 300-600 ms; SMA, 300-600 ms) and mean saccadic-endpoint error during a recording session for
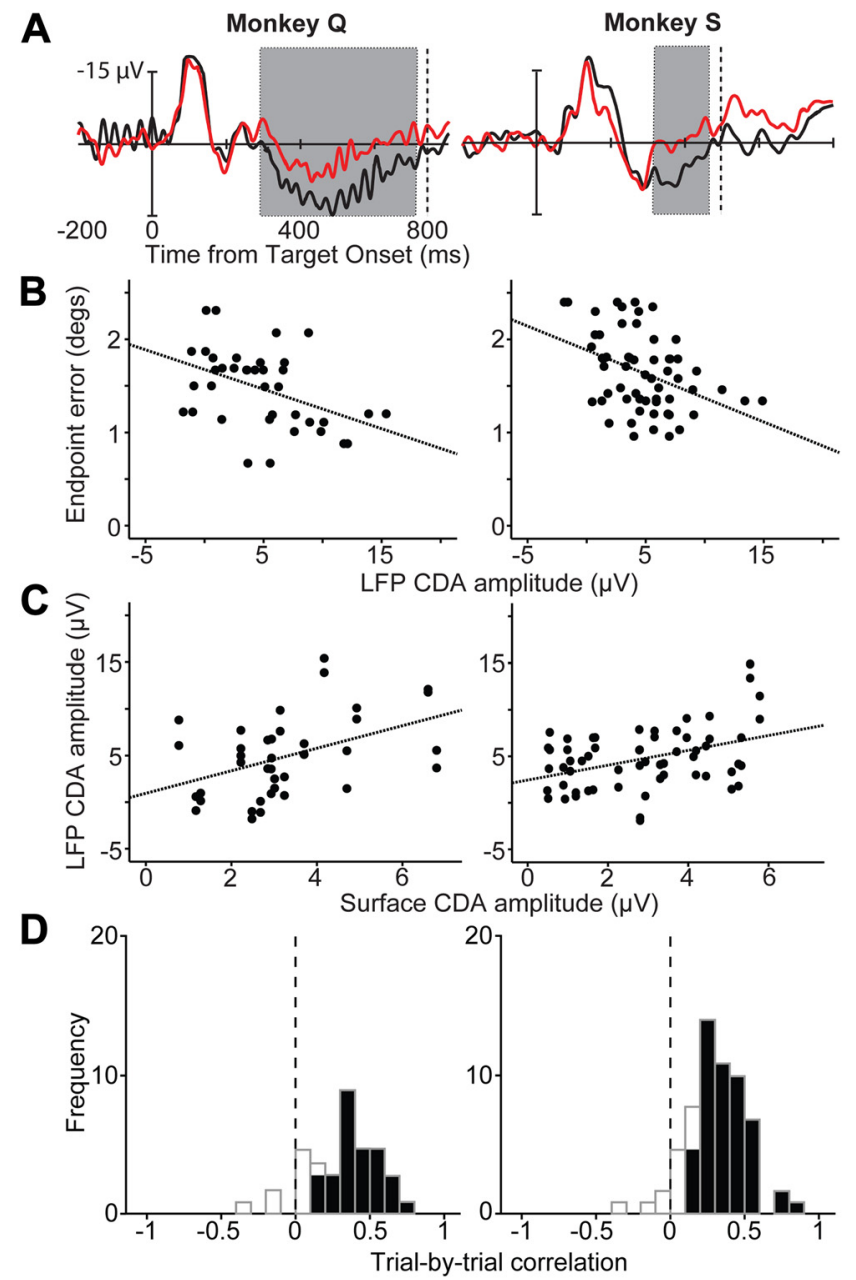

Figure 12. LFP polarizations, LFP and behavior performance relationships, and sessionwise and trialwise LFP-ERP correlations in monkeys $Q$ and $S$. $A$, Grand average LFPs from FEF timelocked to the memory-target onset with the ipsilateral (red) versus contralateral (black) potentials for monkeys $Q$ and $S$. The gray-shaded zones indicate the period during which ipsilateral and contralateral waveforms significantly diverged (i.e., CDA component), and the broken vertical line marks the time subjects were cued to respond. Despite different memory retention intervals ( $Q, 750 \mathrm{~ms}$, and $S, 500 \mathrm{~ms}$ after memory target onset), both monkeys $Q$ and $S$ showed significantly sustained LFP polarizations during the delay period. B, Scatterplots showing significant negative linear correlations between mean variable error (in degrees of visual angle) and mean surface CDA amplitude (ipsilateral minus contralateral waveforms) are illustrated for both monkeys (Q: $r=-0.441, p<0.01$; S: $r=-0.408, p<0.01)$. C, Scatterplots of significant positive linear correlations between mean LFP CDA amplitude in FEF and mean surface CDA amplitude over posterior electrode sites are illustrated on a session-by-session basis for monkeys $Q(r=0.445 ; p<0.01)$ and $S(r=0.409 ; p<0.01)$. D, Trial-by-trial correlations between mean LFP CDA amplitude in FEF and mean surface CDA amplitude over posterior cortex are illustrated for monkeys $Q$ (mean $r \pm$ SE, $0.307 \pm 0.041 ; 29$ of 38 LFP-ERP pairs) and $S(0.309 \pm 0.027 ; 50$ of 62 LFP-ERP pairs). Significant correlation coefficients are represented by the dark bars, and the dashed vertical lines indicate a correlation of zero.

$\operatorname{FEF}\left(r_{(99)}=-0.415 ; p<0.01\right)$ and $\operatorname{SEF}\left(r_{(65)}=-0.372 ; p<\right.$ $0.01)$, but not for SMA $\left(r_{(77)}=-0.036 ; p=0.757\right)$. Mirroring the pattern observed with the CDA, this shows that the greater the amplitude of the sustained negativity in the LFPs, the more precise was the behavioral report of the remembered location.

If the frontal areas we recorded from contribute to the generation of the CDA measured over posterior cortex, then the amplitude of the CDA should covary with the LFP polarization in these cortical areas. We computed the correlations between mean CDA amplitude and the LFP amplitudes during the memory retention intervals in FEF (300-500 ms), SEF (300-600 ms), and 

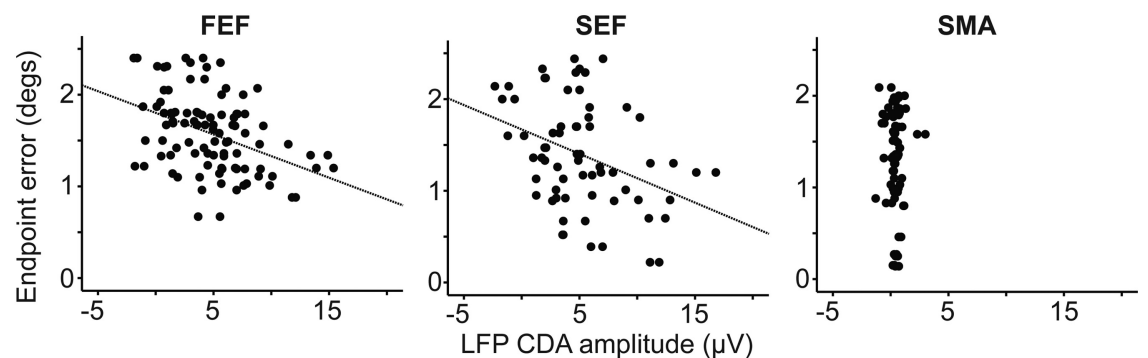

Figure 13. The relationship between monkey intracranial LFP amplitude and behavioral performance. Scatterplots of mean saccadic endpoint error (variable error in degrees of visual angle) against mean LFP amplitude (contralateral minus ipsilateral) are shown on a session-by-session basis.

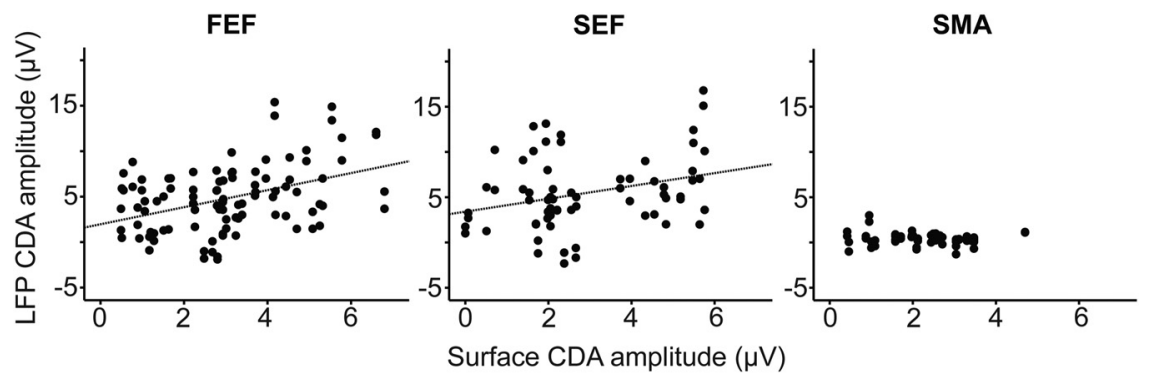

Figure 14. The relationship between monkey intracranial LFP amplitude and surface ERP across sessions. Scatterplots of mean LFP amplitude against mean surface CDA amplitude are illustrated on a session-by-session basis.

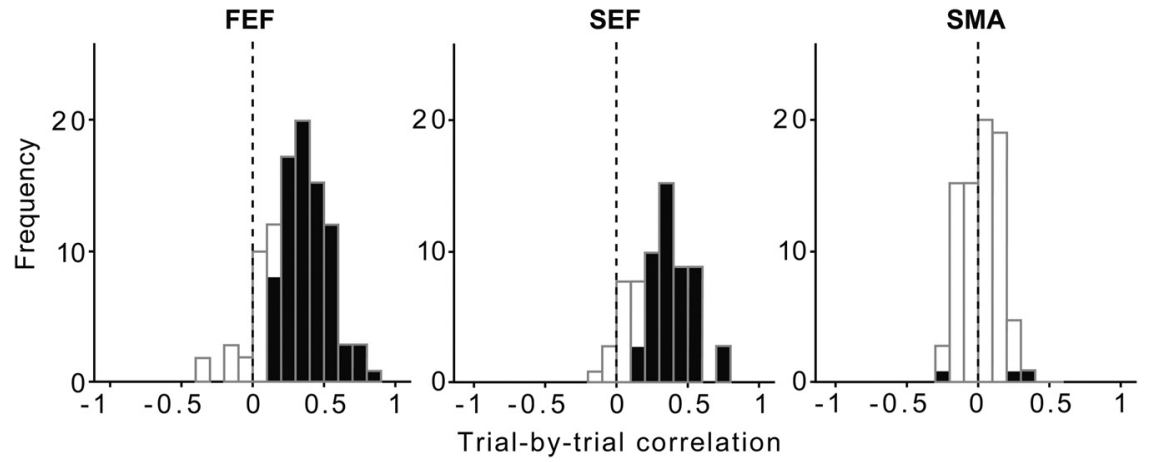

Figure 15. The relationship between the amplitude of the monkey intracranial LFP and surface ERP across trials. Trial-by-trial correlations between mean LFP amplitude and mean surface CDA amplitude were significant for a majority of recording sites in FEF and SEF, but not in SMA. Significant correlation coefficients are illustrated by dark bars, and the dashed vertical lines indicate a correlation of zero.
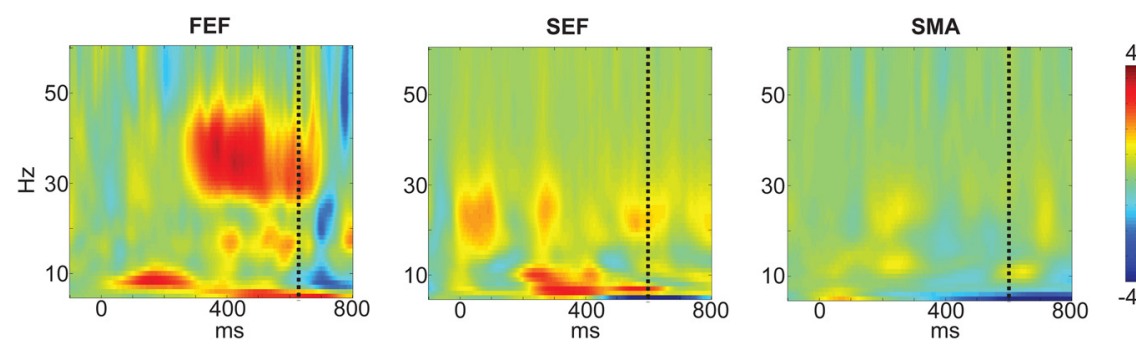

Figure 16. Time-frequency total power plots of lateralized intracranial LFPs. Grand average LFP power data from recordings sites in FEF, SEF, and SMA are illustrated as the difference between power for right and left hemifield stimuli collapsed across right and left hemispheric recording sites, time-locked to memory item onset. The dashed vertical lines mark the time subjects were cued to respond.

Similarly, amplitude relationships between the lateralized LFPs and the CDA on a trial-by-trial basis were significant (Fig. 15; Fisher's $z$ test, $p<0.05$ ) for an overwhelming majority of LFP-ERP pairs in FEF (mean $r \pm \mathrm{SE}, 0.309 \pm 0.023$; 74 of 100 LFP-ERP pairs) and SEF (0.300 \pm 0.025; 49 of 66 LFP-ERP pairs), but not in SMA $(0.019 \pm 0.016 ; 3$ of 78 LFP-ERP pairs; Fig. 12D).

Next, we compared the onset latency of the memory-related effects in the intracranial LFPs and the extracranial ERPs (i.e., the CDA). If the activity in FEF or SEF are feeding back to the posterior areas that appear to generate the CDA as implicated by the topographic analyses, then the lateralized LFPs should onset simultaneously or even before the surface CDA (Cohen et al., 2009). Using the same statistical criteria, we found that the mean onset of the FEF LFPs $(310 \pm 11 \mathrm{~ms})$ was not significantly different from the onset of the concurrently recorded surface CDA $(337 \pm 11 \mathrm{~ms})\left(F_{(1,99)}=1.939 ; p=0.167\right)$, whereas the mean latency of the lateralized LFP in SEF (267 $\pm 17 \mathrm{~ms})$ preceded the onset of the CDA recorded at the surface $\left(F_{(1,65)}=19.584 ; p<0.01\right)$. Finally, we assessed the frequency content of the LFPs in the frontal regions during memory retention. Given that theta and gamma oscillations are linked to working memory processes (Lisman, 2010), the presence or absence of these frequencies in the frontal structures under study should provide an additional line of converging evidence that the activity in these regions underlies memory maintenance. Time-frequency analysis of delay period LFPs revealed prominent lateralized rhythmic activity in the theta $(4-8 \mathrm{~Hz})$ and gamma bands $(30-50 \mathrm{~Hz})$ in FEF, the theta band $(4-8 \mathrm{~Hz})$ in SEF, but no sustained peaks in LFP power spectra were observed in SMA (Figs. 16, 17). As shown in Table 1, statistical analysis confirmed that significant lateralized oscillations were observed in FEF and SEF, but not SMA. These results reveal the oscillatory character of the frontal regions under investigation and confirm the involvement of FEF and SEF while further ruling out SMA in generating the visuospatial working memory effects observed at the surface electrodes. The presence of higher frequency activity in the intracranial recordings relative to the low-frequency modulations during memory mainte-

SMA (300-600 ms). The correlations between CDA amplitude and LFP amplitude across the recordings were significant in FEF $\left(r_{(99)}=0.419 ; p<0.01\right)$ and $\operatorname{SEF}\left(r_{(65)}=0.302 ; p<0.02\right)$, but not in SMA $\left(r_{(77)}=-0.103 ; p=0.367\right)$ (Fig. 14) (see also Fig. 12C). nance on the surface electrodes is consistent with previous studies showing that the skull serves as a low-pass filter that sharply attenuates activity in the gamma band in EEG and ERP recordings (Cooper et al., 1965; Nunez and Srinivasan, 2006). 

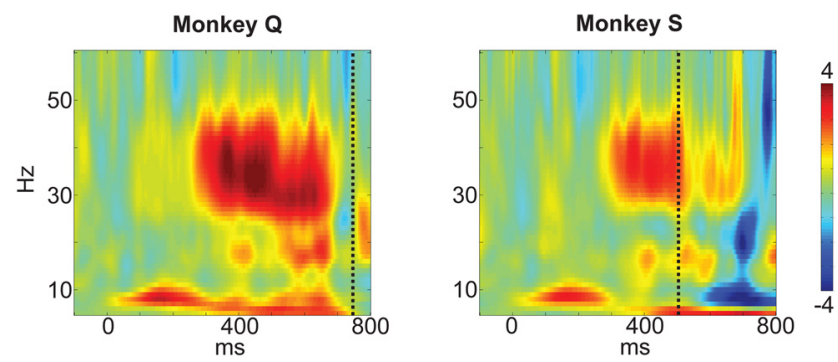

Figure 17. Time-frequency total power plots of lateralized intracranial LFPs in monkeys $Q$ and S. Grand average LFP power data from recordings sites in FEF are illustrated as the difference between power for right and left hemifield stimuli collapsed across right and left hemispheric recording sites for monkeys $\mathrm{Q}$ and $\mathrm{S}$, time-locked to memory item onset.

Table 1. Statistical analyses of the lateralized delay period LFP power in the theta and gamma bands across frontal areas and monkeys

\begin{tabular}{lllllll}
\hline & \multicolumn{2}{l}{ Theta $(4-8 \mathrm{~Hz})$} & & \multicolumn{2}{l}{ Gamma $(30-50 \mathrm{~Hz})$} \\
\cline { 2 - 3 } Area, hemisphere, monkey & $t$ & & & & & \\
FEF, left, Q & $t_{(37)}=2.50$ & $p<0.02$ & & $t_{(37)}=3.60$ & $p<0.001$ \\
FEF, right, Q & $t_{(37)}=2.14$ & $p<0.03$ & & $t_{(37)}=3.53$ & $p<0.002$ \\
FEF, left, S & $t_{(61)}=2.11$ & $p<0.05$ & & $t_{(61)}=2.39$ & $p<0.02$ \\
FEF, right, S & $t_{(61)}=2.25$ & $p<0.04$ & & $t_{(61)}=2.51$ & $p<0.02$ \\
SEF, right, F & $t_{(65)}=2.46$ & $p<0.02$ & & $t_{(65)}=-0.54$ & $p=0.59$ \\
SMA, right, Z & $t_{(77)}=0.79$ & $p=0.43$ & & $t_{(77)}=0.64$ & $p=0.53$ \\
\hline
\end{tabular}

\section{Discussion}

By taking the rare step of recording surface ERPs from humans and monkeys performing the same task (Arthur and Starr, 1984), we discovered a macaque homolog of the human CDA component indexing visuospatial working memory maintenance. The monkey CDA satisfied multiple criteria for establishing homology, including timing, spatial distribution across the skull, scaling with cognitive demands (i.e., the length of delay), underlying oscillatory activity, and its relationship to behavioral performance. This homology links the scalp potentials recorded from macaques and humans during visuospatial working memory maintenance, bridging an empirical gap between two disparate literatures in neuroscience (Gnadt and Andersen, 1988; Funahashi et al., 1989; Colby et al., 1996; Constantinidis and Steinmetz, 1996; Chafee and Goldman-Rakic, 1998; Constantinidis et al., 2002; Pesaran et al., 2002; Vogel and Machizawa, 2004; Wang et al., 2011). Our findings validate the macaque as a model of human visuospatial working memory maintenance and allow us to draw stronger conclusions about the neural mechanisms of humans from the previous electrophysiological studies of monkeys.

Our findings show that the LFPs in FEF and SEF, but not in SMA, contribute to the generation of the CDA indexing working memory maintenance. This conclusion is motivated by four aspects of the results. First, the amplitude of the delay period LFPs in FEF and SEF predicted the amplitude of the CDA measured at the surface ERP electrodes. Second, the absence of similar effects in SMA rules out the hypothesis that the local fields generated in this area contribute to the surface ERP component. This observation is also important because it shows that the effects in the more frontal regions are not simply due to volume conduction, as SMA lies closer to the posterior focus of the CDA than FEF or SEF. The lack of theta and gamma oscillations in SMA provide further converging evidence that rules out this area as a contributor to the surface effects as activity in these frequency bands are known to play a key role in physiological processes important for working memory (Lisman, 2010). Third, the mnemonic LFPs in FEF and SEF were coincident with or preceded the onset of the surface CDA. Fourth, the FEF and SEF LFPs correlated with behavioral accuracy, just as did the CDA recorded over posterior cortex. These findings show that the frontal areas we recorded from are part of a distributed neural network that underlies the CDA and demonstrates the utility of concurrent ERP and invasive microelectrode recordings.

The participation of SEF in CDA generation deserves further mention. Despite much overlap in neuroanatomy, physiology, and function, this agranular frontal region differs from FEF in multiple respects, including density and topography of its projections to oculomotor structures, connectivity to other frontal structures, and visuospatial encoding (Johnston and Everling, 2011). Furthermore, recent research demonstrates that SEF is more concerned with executive control settings than the direct control of attention or saccade production (Stuphorn and Schall, 2006). Our findings also demonstrate a role for SEF in an important aspect of executive functioning, the control of visuospatial working memory maintenance.

As evidence accumulates demonstrating that the CDA is a reliable electrophysiological marker of working memory maintenance (Vogel and Machizawa, 2004; Vogel et al., 2005; Woodman and Vogel, 2008; Carlisle et al., 2011), it is important to understand its neural origins (Perez and Vogel, 2012). This is motivated by evidence showing that working memory deficits occur in numerous disorders, including Alzheimer's disease, attention deficit/hyperactivity disorder, and most prominently schizophrenia (Green et al., 2000; Braver et al., 2002), with the CDA holding promise as a diagnostic tool in clinical research (Perez and Vogel, 2012). The intraparietal sulcus is a likely contributor to the CDA in humans, consistent with the dorsal, posterior scalp distribution and role in working memory storage (Todd and Marois, 2004; Xu and Chun, 2006). However, it is not possible to infer the generators of an ERP component simply based on its scalp distribution (Urbach and Kutas, 2002), and the present findings demonstrate that neural activity can be clearly measured in brain areas that is not observed at electrodes directly above on the skull. This is consistent with the expectation that ERP components are unlikely to be generated by a single cortical source (Luck, 2005; Nunez and Srinivasan, 2006). The intracranial findings reported here rule out the hypothesis of a single source.

Our intracranial results support the hypothesis that prefrontal cortex (FEF) plays a significant role in the circuit generating the CDA (Fuster and Alexander, 1971; Goldman-Rakic, 1987; Funahashi et al., 1989) and extends our knowledge by implicating a primarily visuomotor and cognitive control region (SEF) and excluding a part of the sensorimotor cortex (SMA). Presumably, the electrical fields generated in FEF and SEF are either actively canceled or of such a geometry that they do not result in a more frontally skewed CDA across the surface electrodes (Fig. 4C,D). One might expect that the presence of ipsilateral receptive fields in the prefrontal regions of FEF and SEF might be the cause of the lateralized CDA being observed above such areas (Schall, 1991a,b). However, our LFP recordings show that lateralized memory-related field potentials are evident in these cortical regions, even if the geometry of these electrical fields does not visibly contribute to the distribution of the CDA cross the head. It seems likely, given the timing of the intracranial effects, that the frontal regions we recorded from here feedback to posterior regions, like parietal areas and even lower-level areas of extrastriate and striate cortex (Fig. 4), with this feedback contributing to the generation of the local fields in posterior visual areas that account 
for the observed CDA distribution at the surface. At this point, it is unclear whether the nature of the neural activity underlying the $\mathrm{CDA}$ is that of sustained firing of neurons, oscillatory enhancement, or relative suppression. However, these are network-dynamic hypotheses that future concurrent intracranial recordings will be able to test in further detail.

Future work using our combination of ERP and intracranial recordings will be aimed at distinguishing between competing models of visuospatial working memory that differ in their proposed roles that attention mechanisms play in the maintenance of information. For example, one theoretical perspective is that information is maintained in visuospatial working memory by the deployment of attention to the remembered location in the memory-guided saccade task (Cowan, 1999; Awh and Jonides, 2001). This could explain why the lateralized ERP effects of covert attentional deployment resemble those of working memory maintenance reported here at the level of the surface ERPs (Woodman et al., 2007; Cohen et al., 2009). However, other theories propose that the maintenance of information and covert attentional selection rely upon separate mechanisms (Baddeley, 2003). The set of empirical tools that we have developed here hold new promise for definitively distinguishing between cognitive models of working memory. In addition, our findings will guide inactivation and microstimulation studies that will be able to provide causal evidence that characterizes the precise neuroanatomical loci and neurophysiological events that give rise to the macaque homolog of the human CDA.

\section{References}

Arthur DL, Starr A (1984) Task-relevant late positive component of the auditory event-related potential in monkeys resembles P300 in humans. Science 223:186-188.

Awh E, Jonides J (2001) Overlapping mechanisms of attention and spatial working memory. Trends Cogn Sci 5:119-126.

Baddeley A (2003) Working memory: looking back and looking forward. Nat Rev Neurosci 4:829-839.

Bahill AT, McDonald JD (1983) Smooth pursuit eye movements in response to predictable target motions. Vision Res 23:1573-1583.

Braver TS, Cohen JD, Barch DM (2002) The role of the prefrontal cortex in normal and disordered cognitive control: a cognitive neuroscience perspective. In: Principles of frontal lobe function (Stuff DT, Knight RT, eds), pp 428-448. Oxford: Oxford UP.

Carlisle NB, Arita JT, Pardo D, Woodman GF (2011) Attentional templates in visual working memory. J Neurosci 31:9315-9322.

Chafee MV, Goldman-Rakic PS (1998) Neuronal activity in macaque prefrontal area $8 \mathrm{a}$ and posterior parietal area 7ip related to memory guided saccades. J Neurophysiol 79:2919-2940.

Clark VP, Fan S, Hillyard SA (1995) Identification of early visual evoked potential generators by retinotopic and topographic analyses. Hum Brain Mapp 2:170-187.

Cohen JY, Heitz RP, Schall JD, Woodman GF (2009) On the origin of eventrelated potentials indexing covert attentional selection during visual search. J Neurophysiol 102:2375-2386.

Colby CL, Duhamel JR, Goldberg ME (1996) Visual, presaccadic, and cognitive activation of single neurons in monkey lateral intraparietal area. J Neurophysiol 76:2841-2852.

Constantinidis C, Steinmetz MA (1996) Neuronal activity in posterior parietal area 7a during the delay periods of a spatial memory task. J Neurophysiol 76:1352-1355.

Constantinidis C, Williams GV, Goldman-Rakic PS (2002) A role for inhibition in shaping the temporal flow of information in prefrontal cortex. Nat Neurosci 5:175-180.

Cooper R, Winter AL, Crow HJ, Walter WG (1965) Comparison of subcortical, cortical, and scalp activity using chronically indwelling electrodes in man. Electroencephalogr Clin Neurophysiol 18:217-228.

Cowan N (1999) An embedded-processes model of working memory. In: Models of working memory: mechanisms of active maintenance and ex- ecutive control (Miyake A, Shah P, eds), pp 62-101. Cambridge, UK: Cambridge UP.

Cowan N (2001) The magical number 4 in short-term memory: a reconsideration of mental storage capacity. Behav Brain Sci 24:87-185.

Curtis CE, Rao VY, D'Esposito M (2004) Maintenance of spatial and motor codes during oculomotor delayed response tasks. J Neurosci 24:3944-3952.

Donchin E, Coles MGH (1988) Is the P300 component a manifestation of context updating. Behav Brain Sci 11:357-374.

Fuchs M, Kastner J, Wagner M, Hawes S, Ebersole JS (2002) A standardized boundary element method volume conductor model. Clin Neurophysiol 113:702-712.

Funahashi S, Bruce CJ, Goldman-Rakic PS (1989) Mnemonic coding of visual space in the monkey's dorsolateral prefrontal cortex. J Neurophysiol 61:331-349.

Fuster JM, Alexander GE (1971) Neuron activity related to short-term memory. Science 173:652-654.

Gnadt JW, Andersen RA (1988) Memory related motor planning activity in posterior parietal cortex of macaque. Exp Brain Res 70:216-220.

Godlove DC, Emeric EE, Segovis CM, Young MS, Schall JD, Woodman GF (2011) Event-related potentials elicited by errors during the stop-signal task. I: Macaque monkeys. J Neurosci 31:15640-15649.

Goldman-Rakic PS (1987) Circuitry of the prefrontal cortex and the regulation of behavior by representational knowledge. In: Handbook of physiology (Plum F, Mountcastle VB, eds). Bethesda, MD: American Physiological Society.

Green MF, Kern RS, Braff DL, Mintz J (2000) Neurocognitive deficits and functional outcome in schizophrenia: are we measuring the "right stuff?" Schizophr Bull 26:119-136.

Grimault S, Robitaille N, Grova C, Lina JM, Dubarry AS, Jolicoeur P (2009) Oscillatory activity in parietal and dorsolateral prefrontal cortex during retention in visual short-term memory: additive effects of spatial attention and memory load. Hum Brain Mapp 30:3378-3392.

Hanes DP, Schall JD (1995) Countermanding saccades in macaque. Vis Neurosci 12:929-937.

Helmholtz Hv (1853) Ueber einige Gesetze der Vertheilung elektrischer Ströme in körperlichen Leitern mit Anwendung auf die thierischelektrischen Versuche. Annalen der Physik und Chemie 89:211-233, 354-377.

Jennings JR, Wood CC (1976) The e-adjustment procedure for repeatedmeasures analyses of variance. Psychophysiology 13:277-278.

Johnston KE, Everling S (2011) Frontal cortex and flexible control of saccades. In: The Oxford handbook of eye movements (Liversedge S, Gilchrist I, Everling S, eds), pp 279-302. New York: Oxford UP.

Jokisch D, Jensen O (2007) Modulation of gamma and alpha activity during a working memory task engaging the dorsal or ventral stream. J Neurosci 27:3244-3251.

Lamme VA, Van Dijk BW, Spekreijse H (1992) Texture segregation is pressed by primary visual cortex in man and monkey. Evidence from VEP experiments. Vision Res 32:797-807.

Lisman J (2010) Working memory: the importance of theta and gamma oscillations. Curr Biol 20:R490-R492.

Luck SJ (2005) An introduction to the event-related potential technique. Cambridge, MA: MIT.

Luck SJ, Vogel EK (1997) The capacity of visual working memory for features and conjunctions. Nature 390:279-281.

Mazaheri A, Jensen O (2008) Asymmetric amplitude modulations of brain oscillations generate slow evoked responses. J Neurosci 28:7781-7787.

Mazaheri A, Jensen O (2010) Rhythmic pulsing: linking ongoing brain activity with evoked responses. Front Hum Neurosci 4:177.

Nunez PL, Srinivasan R (2006) Electric fields of the brain: the neurophysics of EEG, Ed 2. Oxford: Oxford UP.

Oostenveld R, Fries P, Maris E, Schoffelen JM (2011) FieldTrip: open source software for advanced analysis of MEG, EEG, and invasive electrophysiological data. Comput Intell Neurosci 2011:156869.

Perez VB, Vogel EK (2012) What ERPs can tell us about working memory. In: Oxford handbook of event-related potential components (Luck SJ, Kappenman E, eds), pp 361-372. New York: Oxford UP.

Pesaran B, Pezaris JS, Sahani M, Mitra PP, Andersen RA (2002) Temporal structure in neuronal activity during working memory in macaque parietal cortex. Nat Neurosci 5:805-811.

Ploner CJ, Gaymard B, Rivaud S, Agid Y, Pierrot-Deseilligny C (1998) Tem- 
poral limits of spatial working memory in human. Eur J Neurosci 10:794-797.

Reinhart RM, Carlisle NB, Kang MS, Woodman GF (2012) Event-related potentials elicited by errors during the stop-signal task. II: Human effector specific error responses. J Neurophysiol 107:2794-2807.

Ruchkin DS, Johnson R Jr, Grafman J, Canoune H, Ritter W (1992) Distinctions and similarities among working memory processes: an event-related potential study. Brain Res Cogn Brain Res 1:53-66.

Sauseng P, Klimesch W, Heise KF, Gruber WR, Holz E, Karim AA, Glennon M, Gerloff C, Birbaumer N, Hummel FC (2009) Brain oscillatory substrates of visual short-term memory capacity. Curr Biol 19:1846-1852.

Schall JD (1991a) Neuronal activity related to visually guided saccadic eye movements in the supplementary motor area of rhesus monkeys. J Neurophysiol 66:530-558.

Schall JD (1991b) Neuronal activity related to visually guided saccades in the frontal eye fields of rhesus monkeys: comparison with supplementary eye fields. J Neurophysiol 66:559-579.

Schroeder CE, Tenke CE, Givre SJ, Arezzo JC, Vaughan HG Jr (1991) Striate cortical contribution to the surface-recorded pattern-reversal VEP in the alert monkey. Vision Res 31:1143-1157.

Schroeder CE, Tenke CE, Givre SJ (1992) Subcortical contributions to the surface-recorded flash-VEP in the awake macaque. Electroencephalogr Clin Neurophysiol 84:219-231.

Stuphorn V, Schall JD (2006) Executive control of countermanding saccades by the supplementary eye field. Nat Neurosci 9:925-931.

Todd JJ, Marois R (2004) The capacity limit of visual short-term memory in human posterior parietal cortex. Nature 428:751-754.

Urbach TP, Kutas M (2002) The intractability of scaling scalp distributions to infer neuroelectric sources. Psychophysiology 39:791-808.

Van Der Werf J, Jensen O, Fries P, Medendorp WP (2008) Gamma-band activity in human posterior parietal cortex encodes the motor goal during delayed prosaccades and antisaccades. J Neurosci 28:8397-8405.

van Dijk H, van der Werf J, Mazaheri A, Medendorp WP, Jensen O (2010)
Modulations in oscillatory activity with amplitude asymmetry can produce cognitively relevant event-related responses. Proc Natl Acad Sci U S A 107:900-905.

Vogel EK, Machizawa MG (2004) Neural activity predicts individual differences in visual working memory capacity. Nature 428:748-751.

Vogel EK, McCollough AW, Machizawa MG (2005) Neural measures reveal individual differences in controlling access to working memory. Nature 438:500-503.

Wagner M, Fuchs M, Kastner J (2007) SWARM: sLORETA-weighted accurate minimum norm inverse solutions. International Congress Series 1300:185-188.

Wang M, Gamo NJ, Yang Y, Jin LE, Wang X-J, Laubach M, Mazer JA, Lee D, Arnsten AF (2011) Neuronal basis of age-related working memory decline. Nature 476:210-213.

White JM, Sparks DL, Stanford TR (1994) Saccades to remembered target locations: an analysis of systematic and variable errors. Vision Res 34:79-92.

Woodman GF (2010) A brief introduction to the use of event-related potentials in studies of perception and attention. Atten Percept Psychophys 72:2031-2046.

Woodman GF (2012) Homologues of human event-related potential components in nonhuman primates. In: The Oxford handbook of eventrelated potential components (Luck SJ, Kappenman E, eds), pp 611-625. New York: Oxford UP.

Woodman GF, Vogel EK (2008) Top-down control of visual working memory consolidation. Psychon Bull Rev 15:223-229.

Woodman GF, Kang MS, Rossi AF, Schall JD (2007) Nonhuman primate event-related potentials indexing covert shifts of attention. Proc Natl Acad Sci U S A 104:15111-15116.

Xu Y, Chun MM (2006) Dissociable neural mechanisms supporting visual short-term memory for objects. Nature 440:91-95.

Zhang W, Luck SJ (2008) Discrete fixed-resolution representations in visual working memory. Nature 453:233-235. 